\title{
Tafonomia de moluscos fósseis do Grupo Bauru \\ (Cretáceo Superior, Bacia Bauru), na região do município de Monte Alto, São Paulo, Brasil \\ Taphonomy of fossils mollusks of Bauru Group (Late Cretaceous, Bauru Basin), in Monte Alto municipality region, São Paulo, Brazil
}

\author{
Renato Pirani Ghilardi', Fernando César Paiva D'Ágosta', Kléber Alves', \\ Antonio Celso de Arruda Campos" \\ 'Universidade Estadual Paulista. Bauru, São Paulo, Brasil \\ "Museu Histórico e Paleontológico de Monte Alto. Monte Alto, São Paulo, Brasil
}

\begin{abstract}
Resumo: A tafonomia de fósseis de moluscos bivalves depositados em coleção científica e coletados em afloramento do Grupo Bauru (Cretáceo Superior, Bacia Bauru), da região do municíio de Monte Alto, São Paulo, é analisada neste trabalho. A preservação dos organismos recristalizados em matriz carbonática indica remobilização do substrato por agente energético unidirecional, em momentos de maior vazão fluvial. Os espécimes com valvas conjugadas possuem sedimento interno similar ao externo, implicando uma baixa exposição dos organismos à Zona Tafonomicamente Ativa e sugerindo baixa mistura temporal dos bioclastos. A porção posterior fragmentada de forma truncada, encontrada nos exemplares de coleção científica, possivelmente está relacionada à incapacidade do táxon reescavar o substrato em períodos de seca. Os dois tipos de preservação encontrados corroboram evidências de outra natureza, que indicam paleoambientes fluviais no Grupo Bauru, na área estudada.
\end{abstract}

Palavras-chave: Tafonomia. Bivalves fósseis. Formação Marília. Cretáceo Superior.

Abstract: Bivalves mollusks fossils of Bauru Group (Late Cretaceous, Bauru Basin) deposited in scientific collections and collected in outcrops from Monte Alto municipality, São Paulo, are analyzed in their taphonomy. The preservation of recrystallized individual in carbonatic matrix indicates substrate remobilization by unidirectional energetic event in fluvial discharge. The specimens with conjugated valves possess internal sediment similar to the external indicating low exposition to Taphonomical Active Zone, suggesting a bioclastic low time-averaging. The truncate and fragmented posterior portion of specimens from scientific collections is probably related to the incapacity of the taxa to reburrowing the substrate in drowning periods. Both taphonomic patterns corroborate evidences of a fluvial paleoenvironment in the Bauru Group.

Keywords: Taphonomy. Fossil Bivalves. Marilia Formation. Late Cretaceous.

GHILARDI, R. P., F. C. P. D'ÁGOSTA, K. ALVES \& A. C. A. CAMPOS, 2011. Tafonomia de moluscos fósseis do Grupo Bauru (Cretáceo Superior, Bacia Bauru), na região do município de Monte Alto, São Paulo, Brasil. Boletim do Museu Paraense Emílio Goeldi. Ciências Naturais 6(2): 197-206.

Autor para correspondência: Renato Pirani Ghilardi. Universidade Estadual Paulista Júlio de Mesquita Filho. Faculdade de Ciências de Bauru. Departamento de Ciências Biológicas. Av. Eng. Luiz Edmundo Carrijo Coube, s/n. Bauru, SP, Brasil. CEP 17033-360 (ghilardi@fc.unesp.br). Recebido em 11/05/2010

Aprovado em 04/07/2011

Responsabilidade editorial: Hilton Tulio Costi

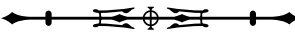




\section{INTRODUÇÃO}

Entre os fósseis do Grupo Bauru (Cretáceo Superior, Bacia Bauru), certamente os mais conhecidos são os crocodilianos, quelônios e dinossauros. Contudo, já foram lá descritos outros macrofósseis, como vegetais, ostracodes, conchostráceos e restos de peixes. São os moluscos, entretanto, o táxon menos estudado do Grupo Bauru, sendo poucos os trabalhos existentes que versam sobre a sua sistemática.

Ihering (1913) foi o primeiro a descrever moluscos bivalves em sedimentos do Grupo Bauru: Itaimbea priscus (Ihering, 1913), coletada em sedimentos arenosos no município de São José dos Dourados (atual São José do Rio Preto), no estado de São Paulo. Somente cerca de 50 anos mais tarde, Arid et al. (1962) descrevem, pela primeira vez, um gastrópode indeterminado procedente de afloramento nas imediações de São José do Rio Preto, no mesmo estado. Mezzalira (1966), Suarez \& Arruda (1968) e Parodiz (1969) aumentam o número de espécies de moluscos descrito para o estado de São Paulo. $\bigcirc$ trabalho de revisão de Mezzalira (1974) é o que descreve a maior quantidade de táxons (?Hydrobia prudentinensis, Viviparus souzai, Physa aridi, Diplodon arrudai, Florenceia peiropolensis, Sancticarolis tolentinoi, Taxodontites paulistanensis, Anodontites freitasi, Anodontites pricei e Monocondylaea cominatoi) conhecidos para o estado de São Paulo. Adicionalmente, Mezzalira (1974), pioneiramente, descreve os moluscos presentes em sedimentos do Grupo Bauru de Goiás e Minas Gerais. No mesmo trabalho, é definida a classificação sistemática dos moluscos do Grupo Bauru da maneira atualmente empregada.

Somente após cerca de vinte anos, Simone \& Mezzalira (1993) voltam ao tema, ao descreverem a preservação de restos de partes moles (palpos, demibrânquias e músculos) em um bivalve Unionoida (Anodontites freitasi) do Grupo Bauru, no estado de São Paulo. Campanha et al. (1993) descrevem o gastrópode ?Hydrobya sp. e Turritella sp. em sedimentos localizados entre Uberaba e Uberlândia, Minas Gerais. Por sua vez, Senra \& Silva e Silva (1999) relatam a ocorrência de gastrópodes (P. aridi e V. souzai) e microbivalves, provisoriamente classificados como pertencentes ao gênero ?Musculium, em sedimentos de Uberaba, Minas Gerais. Em outro trabalho de cunho sistemático, Mezzalira \& Simone (1997) normalizam a situação taxonômica dos gêneros Florenceia, Sancticarolis e Taxodontites já alterados previamente, mas sem valor taxonômico, em Simone \& Mezzalira (1994). Finalmente, Mezzalira \& Simone (1999) descrevem, a partir de material depositado em coleção científica, o gênero Castalia, constituído por duas espécies (C. cretacea e C. minuta), para sedimentos do Grupo Bauru.

A Tabela 1 mostra as espécies de bivalves taxonomicamente válidas do Grupo Bauru, assim como a procedência das mesmas e o tipo de estudo realizado sobre o táxon. É interessante reparar que a região de Monte Claro, no estado de São Paulo, apesar de ser reconhecidamente um importante centro para estudos de vertebrados, não registra nenhuma forma de molusco descrita nos sedimentos ali aflorantes.

Adicionalmente, é notório que os moluscos fósseis do Grupo Bauru carecem de estudos tafonômicos e paleoecológicos mais detalhados e modernos para um melhor entendimento da gênese dessas acumulações. A única exceção aos trabalhos sobre a sistemática destes moluscos é o texto de Ghilardi \& Benedetti (2004) que, de maneira pioneira, tece considerações sobre a ecologia e tafonomia do gênero Anodontites. Os atributos tafonômicos ou assinaturas tafonômicas das ocorrências fossiliferas estão intimamente associados às taxas de sedimentação, além da energia do meio. Assim, com a compreensão desses processos de preservação, os ambientes deposicionais do Grupo Bauru podem ser mais facilmente interpretados.

\section{CONTEXTO GEOLÓGICO}

A Bacia Bauru é a mais extensa sequência sedimentar de idade cretácea da América do Sul, sendo considerada uma entidade tectônica distinta da Bacia do Paraná (Coimbra \& Fernandes, 1994; Fernandes \& Coimbra, 1996). Ocorre na maior parte do planalto ocidental de São Paulo, aflorando também no Triângulo Mineiro, sul de Goiás, norte do 
Tabela 1. Afloramentos relacionados aos táxons fósseis.

\begin{tabular}{|c|c|c|}
\hline Município do afloramento & Espécies descritas & Tipo de estudo realizado \\
\hline Fernando Prestes - SP & Itaimbea priscus & Taxonômico \\
\hline Madeira - GO & Viviparus souzai & Taxonômico \\
\hline Pacaembu Paulista - SP & $\begin{array}{c}\text { Anodontites freitasi, Monocondylaea } \\
\text { cominatoi, Taxodontites paulistanensis, } \\
\text { Castalia cretacea }\end{array}$ & Taxonômico e Morfologia Funcional \\
\hline Peirópolis - MG & Anodontites pricei, Musculium? & Taxonômico \\
\hline Presidente Prudente - SP & $\begin{array}{c}\text { Diplodon arrudai, Anodontites paulistanensis, } \\
\text { Hydrobia prudentinensis }\end{array}$ & Taxonômico \\
\hline São Carlos - SP & Sancticarolis tolentinoi & Taxonômico \\
\hline São José do Rio Preto - SP & Physa aridi & Taxonômico \\
\hline Uberaba - MG & $\begin{array}{c}\text { Florenceia peiropolensis, Hydrobia sp., } \\
\text { Turritelídeos }\end{array}$ & Taxonômico \\
\hline
\end{tabular}

Paraná e sudeste do Mato Grosso (Petri \& Fulfaro, 1983; Manzini, 1996) (Figura 1).

O Grupo Bauru é caracterizado como um pacote sedimentar largamente oxidado (Castro et al., 1999), constituído litologicamente por arenitos e siltitos depositados em ambiente fluvial (Batezelli et al., 2003; Batezelli et al., 2007). A estratigrafia da Bacia Bauru continua controversa e ainda carece de estudos mais aprofundados (Fulfaro \& Perinotto, 1996), sendo interpretada, em geral, como uma associação de diferentes fácies sedimentares, predominantemente continentais, interdigitadas. A Figura 2 sintetiza o conhecimento estratigráfico atual nos estados onde a Bacia Bauru foi mapeada.

Tão controversa quanto a estratigrafia do Grupo Bauru é a determinação da sua idade. Logo após a descrição dos primeiros fósseis, surgiram dúvidas quanto à sua real datação. Atualmente, considera-se o Grupo Bauru como sendo do Cretáceo Superior, devido à presença de dinossauros que ocorrem associados aos moluscos dulcícolas fósseis, cujas extinções ocorreram no mesmo período (Von Huene, 1927; Price, 1953; Arid \& Vizzoto, 1963; Suarez, 1969).

Ambientalmente, os depósitos sedimentares do Grupo Bauru são tipicamente fluviais e lacustres. Durante o ciclo inicial de deposição do Grupo Bauru (formações Caiuá e Santo Anastácio), as fácies representam frentes deltaicas e planícies aluviais. O clima, ao menos para a formação Caiuá, seria árido a semi-árido com presença de dunas e sistema lacustre efêmero interdunas (Popp \& Bigarella, 1975). Já a Formação Adamantina teria sistemas deposicionais mais energéticos, sendo representada por sedimentos transportados por rios de maior porte que os da fase anterior. Ainda nessa formação, encontram-se sedimentos representativos de ambientes de planícies de inundação, associados a sedimentos de canais fluviais. $\bigcirc$ clima seria quente e úmido, alternado por períodos mais secos. Finalmente, a Formação Marília reflete ambiente fluvial meandrante, que grada progressivamente para um sistema fluvial anastomosado, devido ao aumento da aridez e desertificação (Popp \& Bigarella, 1975).

\section{MATERIAIS E MÉTODOS}

\section{AFLORAMENTOS DE COLETA}

O afloramento de Água Limpa (Manzini, 1996), relacionado à Formação Marília, onde os fósseis desse estudo foram coletados, localiza-se nos arredores da cidade de Monte

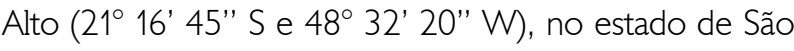
Paulo. De maneira geral, o afloramento é constituído por um pacote com cerca de 113 m de espessura, sendo sua base constituída por $27 \mathrm{~m}$ de arenitos avermelhados, finos 


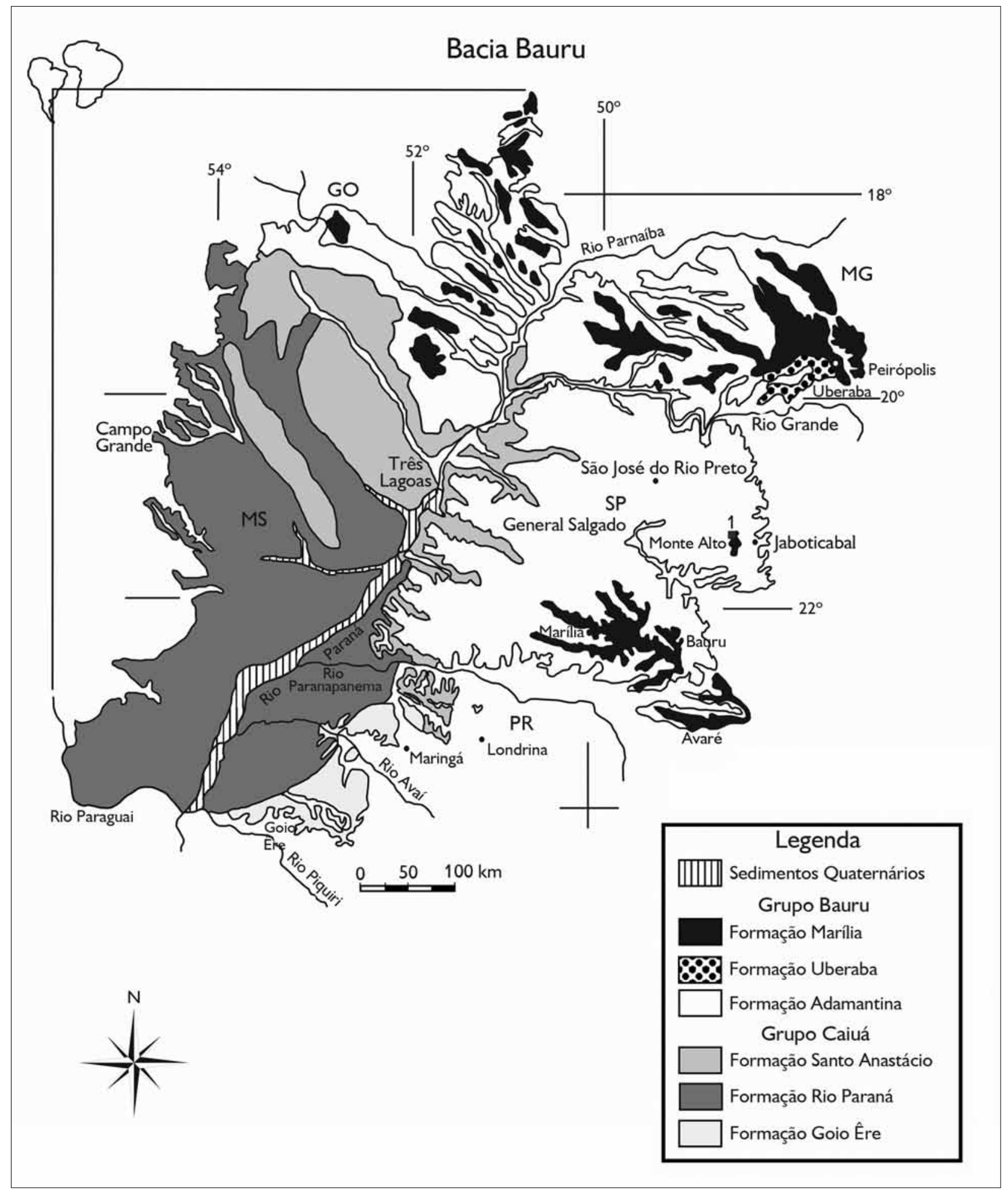

Figura 1. Mapa litoestratigráfico da parte Oriental da Bacia Bauru. 1 - Afloramento Água Limpa, município de Monte Alto, SP. Modificado de Marinho et al. (2006).

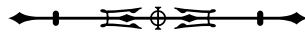




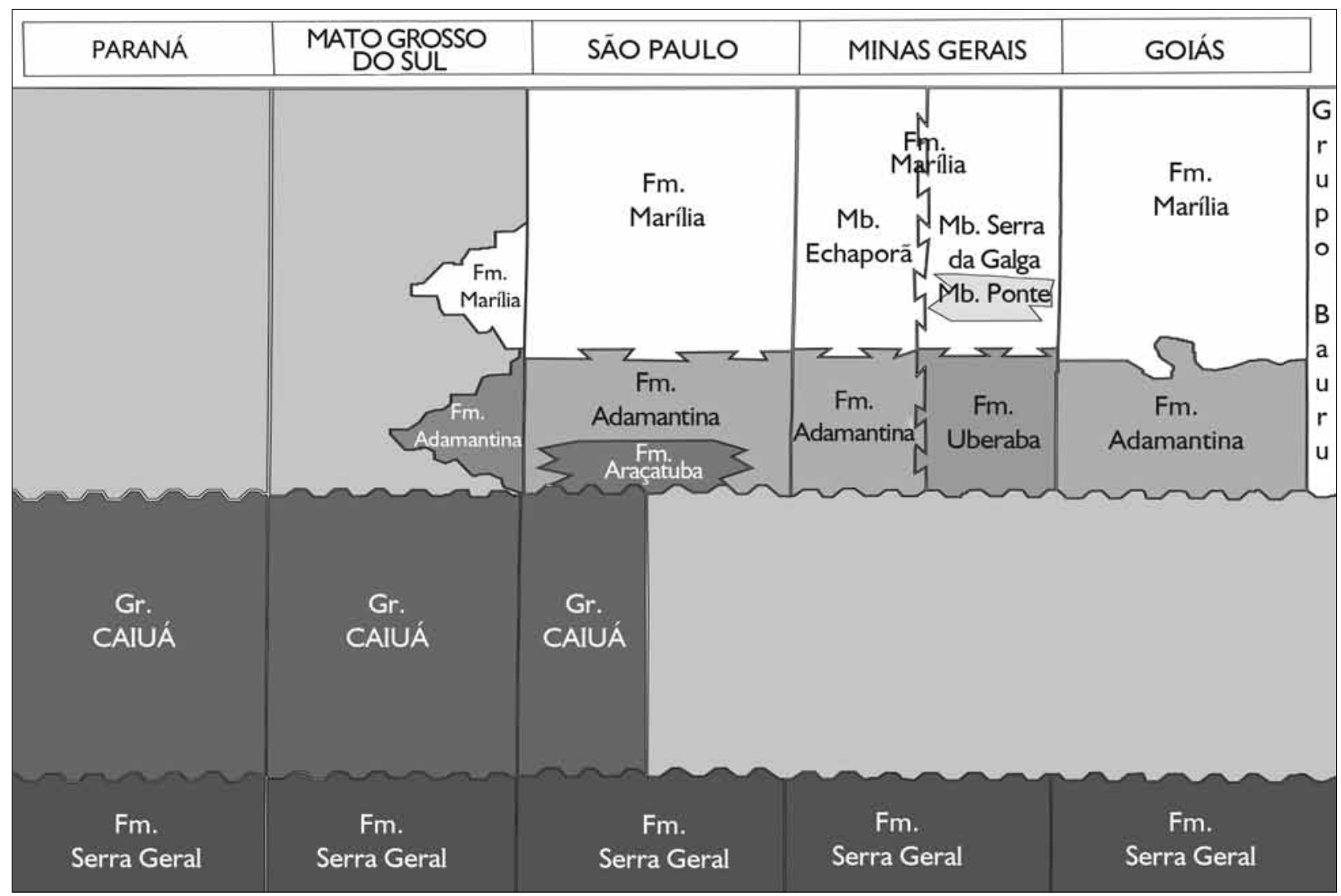

Figura 2. Coluna litoestratigráfica do Grupo Bauru. Modificado de Fulfaro e Perinotto (1996). Gr - Grupo; Fm - Formação; Mb - Membro.

a médios, maciços. A esse pacote é sobreposto arenito vermelho, com raras intercalações de níveis argilosos de coloração castanha. No topo da sequência, ocorrem camadas de arenitos vermelho-esbranquiçados, finos a médios, fortemente carbonáticos, maciços, intercalados a lentes de lamitos avermelhados. Por vezes, esse arenito é intercalado com nódulos carbonáticos, que conferem à rocha um caráter conglomerático. A base da seção possui fragmentos de ossos de dinossauros, enquanto no topo ocorre um nível arenítico, de 0,30 m de espessura, com fósseis de moluscos (Manzini, 1996) (Figura 3).

\section{METODOLOGIA DE COLETA}

Segundo Simões \& Ghilardi (2000), a mistura temporal e espacial entre fósseis é um dos principais fatores que podem conduzir a erros em análises paleoambientais. $\bigcirc$ protocolo tafonômico proposto por Simões \& Ghilardi (2000) padroniza alguns dos procedimentos de campo e laboratório, podendo ser adaptado segundo o escopo da pesquisa e escala de análise. No caso do Grupo Bauru da região de Monte Alto, não há níveis preferenciais de moluscos pelas seções. Prefere-se, assim, nesse trabalho, uma variação na utilização de quadrículas de coleta, como explicado abaixo.

Cada quadrícula corresponde a um volume de rocha, extraído do afloramento, com dimensões de três metros de largura, dois metros de altura e $20 \mathrm{~cm}$ de profundidade, compreendendo, assim, um total de 1,2 $\mathrm{m}^{3}$. Esse volume de rocha coletada tem que se manter constante, independentemente do posicionamento espacial do afloramento, seja ele uma parede vertical, uma parede inclinada ou mesmo uma superfície horizontal. Vale lembrar que, a princípio, a coleta visa volume de rocha e não período

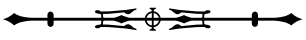


de tempo de deposição, pois, muitas vezes, os estratos representam superfícies temporalmente condensadas.

Para as análises tafonômicas, a descrição das assinaturas bioestratinômicas seguirá basicamente a proposta de Simões \& Kowalewski (1998), incluindo, entre outros: a) a orientação dorso ventral dos bioclastos, isto é, se eles estão com a convexidade para cima ou para baixo, em relação ao substrato; b) a orientação azimutal do maior eixo do bioclasto, para análises de distribuição preferencial dos bioclastos e c) comprimento do maior eixo dos bioclastos. Estes dados, em associação com outros de natureza sedimentológica (e.g., estruturas sedimentares, grau de empacotamento, contatos estratigráficos, geometria da concentração fossilifera, índice de bioturbação) (Kidwell et al., 1986; Kidwell \& Holland, 1991), possibilitam o reconhecimento dos padrões de preservação dos moluscos do Grupo Bauru no afloramento estudado, bem como a determinação de fatores abióticos e bióticos (veja Fürsich \& Oschmann, 1993) responsáveis pela gênese das concentrações fossilíferas. Os dados morfofuncionais seguem a nomenclatura de Stanley (1970) e Ghilardi \& Simões (2000, 2002).

Os cerca de 40 espécimes fósseis coletados nos blocos retirados do afloramento Água Limpa, depois de tratados e analisados, foram depositados na coleção científica do Laboratório de Paleontologia de Macroinvertebrados (LAPALMA), da Universidade Estadual Paulista "Júlio de Mesquita Filho" de Bauru, São Paulo. Cinco exemplares depositados na coleção do Museu de Paleontologia de Monte Alto, sob o número MPMA 1170, coletados em camadas cronocorrelatas, também foram utilizados para as considerações.

\section{RESULTADOS}

A matriz carbonática dificulta a retirada dos organismos dos blocos (Figura 4). A análise dos espécimes, dessa maneira, torna-se dificultada pelas condições genéticas onde, muitas vezes, encontram-se feições secundárias geradas pelo tectonismo da área (Manzini, 1996).

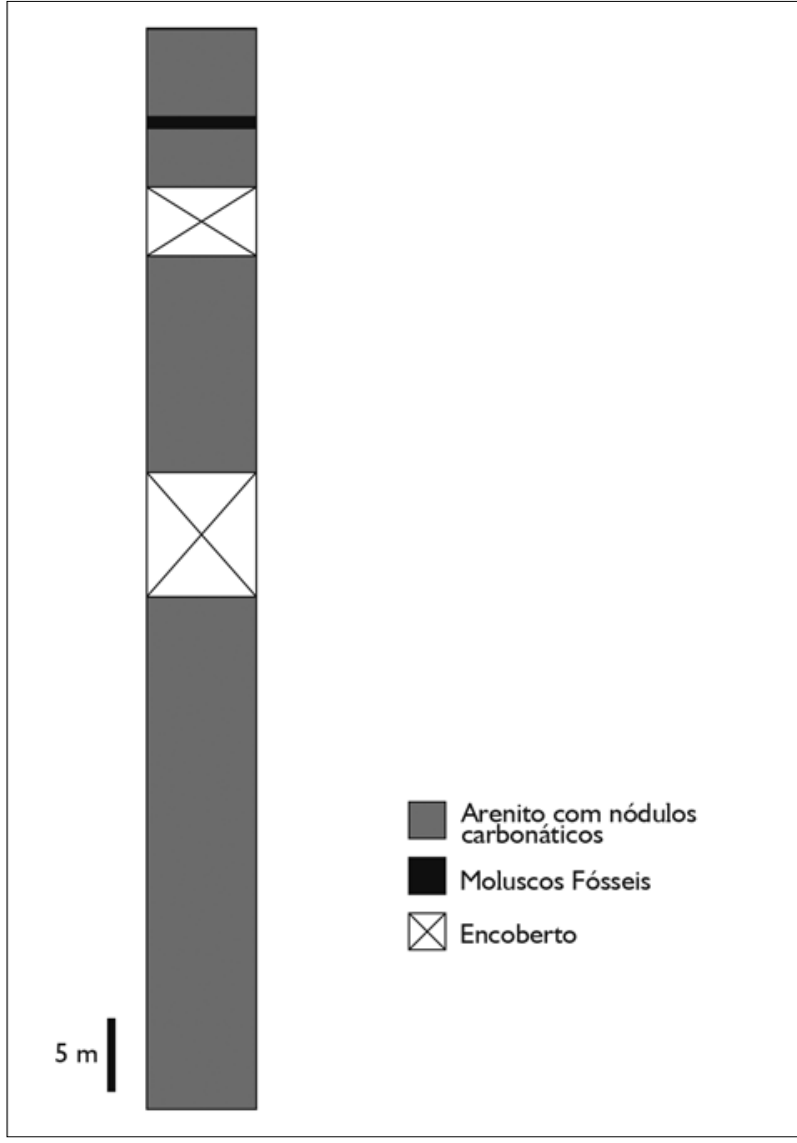

Figura 3. Litoestratigrafia do afloramento Água Limpa, Formação Marília, Grupo Bauru. Modificado de Manzini (1996).

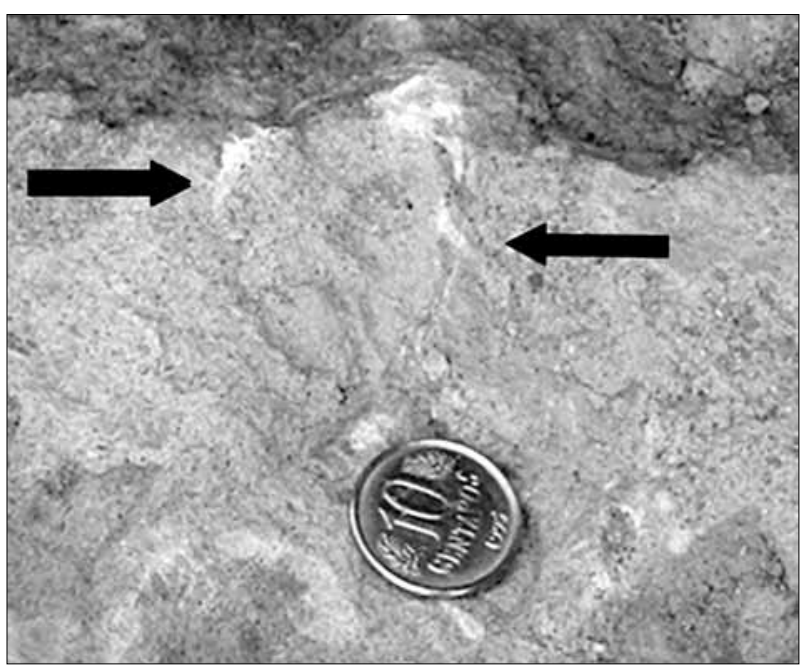

Figura 4. Restos externos de conchas de bivalves (setas) indeterminados, cristalizados do afloramento de Água Limpa, município de Monte Alto, SP.

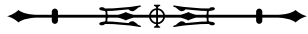


Portanto, os fósseis do afloramento Água Limpa não puderam ser classificados sistematicamente devido às condições de preservação encontradas.

A maioria dos exemplares (85\%) está articulada fechada. Contudo, todas as conchas apresentam-se fora de posição de vida, paralelas ao plano de acamamento. Todos os espécimes encontram-se recristalizados no arenito (Figura 4). As conchas estão caoticamente dispersas pela matriz, não possuindo nível preferencial de preservação na rocha. Um montante de $54 \%$ das conchas desarticuladas possui concavidade para cima. Não há níveis preferenciais desses invertebrados e, quando são encontrados, estão fracamente empacotados pela matriz, prejudicando qualquer tipo de análise mais acurada. Ademais, os exemplares não apresentam sinais de fragmentação, erosão ou dissolução, indicando, provavelmente, pouco transporte pós-morte e rápido soterramento, com pouca exposição na interface água/sedimento.

Por outro lado, os exemplares da coleção do Museu de Paleontologia de Monte Alto (MPMA 1770) apresentam uma particularidade em comum: todos possuem a porção posterior da concha truncada de forma retilínea (Figura 5). Apesar de se apresentarem articulados fechados, é impossível a análise de seu posicionamento de vida, pois não há indicativo de sua disposição em relação ao plano de acamamento.

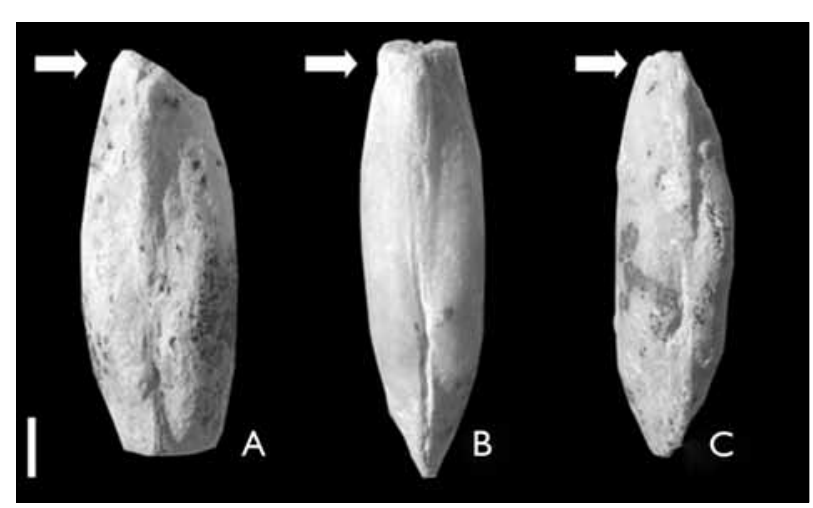

Figura 5. Exemplares de ? Anodontites freitasi (A, B e C) articulados fechados (genericamente catalogados como MPMA 1170). Reparar no padrão de fragmentação na porção posterior da concha (setas). Escala $=1 \mathrm{~cm}$.

\section{DISCUSSÃO}

Em relação à tafonomia dos bivalves do Grupo Bauru, a ausência de conchas em posição de vida, ao menos nos exemplares coletados em afloramento, denota agentes energéticos de fundo capazes de revolverem o substrato e transportarem os bioclastos. A areia grossa associada e a falta de feições sedimentares de tempestades, como hummockyes e turbiditos (Manzini, 1996), indicam a inexistência de eventos únicos de soterramento para esses organismos. A matriz onde os fósseis são preservados é idêntica à matriz encontrada no interior das conchas dos bivalves articulados (Figura 4). Esse fato denota que as conchas ficaram pouco tempo expostas à zona tafonomicamente ativa (ZTA) antes de sua deposição final.

Dessa maneira, os dados acima expostos, analisados em conjunto, indicam prováveis agentes energéticos unidirecionais influenciando na gênese dessas concentrações fossiliferas. Essas condições são encontradas em ambientes fluviais.

Adicionalmente, a presença de formas articuladas fechadas (exemplares catalogados com o número MPMA 1170) em substrato arenoso indica uma provável autoctonia/ parautoctonia desses espécimes. O fato de todos os exemplares serem provenientes de coleções científicas, sem dados de campo, prejudica uma melhor interpretação tafonômica dos mesmos. Contudo, alguns exemplares apresentam, na porção posterior de suas conchas, um padrão de fragmentação truncado (Figura 5). O padrão não parece estar relacionado aos fatores de coleta dos exemplares, mas sim à sua preservação. $O$ corte retilíneo e de posicionamento posterior observado nos diversos espécimes indica um agente energético capaz de remobilizar o sedimento até, pelo menos, a profundidade de vida dos organismos. Contudo, é de se chamar a atenção para a fragmentação constante e recorrente apenas em organismos articulados fechados, o que demonstra a ação ocorrendo ou ainda com o animal vivo ou morto soterrado em posição de vida.

Associado ao exposto, os estudos de bivalves de água doce nunca receberam a mesma atenção dispensada ao estudo de formas marinhas. Entretanto, características morfológicas

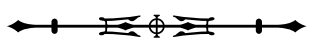


destes organismos são exclusivas (Ghilardi \& Simões, 2002). A carência de estudos se deve, aparentemente, às poucas adaptações exclusivas que os grupos dulcícolas apresentam, assim como seus padrões evolutivos pouco complexos (Savazzi \& Peiyi, 1992). Duarte et al. (1996) chamam a atenção ao fato de que bivalves dulcícolas do Lago Suzana respondem ativamente ao recuo da margem do lago em épocas de seca e em relação ao substrato. De fato, Tevesz \& McCall (1979) já demonstram que os bivalves dulcícolas não respondem tão especificamente ao substrato como os bivalves marinhos. Aparentemente, suas respostas adaptativas estão relacionadas à velocidade do fluxo de água, o que pode retratar, de forma mais fidedigna, seus padrões de distribuição (Huehner, 1987). Infelizmente, os bivalves do Grupo Bauru analisados em coleção científica não apresentam o registro de sua litologia ou de sua distribuição espacial na rocha. Contudo, o fato de eles apresentarem um padrão de fragmentação especíico pode indicar uma distribuição espacial relacionada ao fluxo de água e consequente erosão esporádica sofrida pelo substrato de vida desses moluscos.

Dessa maneira, os espécimes aqui analisados estão mais relacionados à preservação em calhas de rios, onde os bioclastos maiores acumulam-se no substrato arenoso. De fato, o ambiente de deposição do Grupo Bauru sempre foi controverso. A maioria dos trabalhos versa sobre ambiente dulcícolas para essa formação geológica (Petri \& Fulfaro, 1983). A diferenciação, contudo, entre os subambientes fluvial e lacustre para as diferentes formações litológicas do Grupo Bauru sempre foi motivo de debate (veja Mezzalira, 1974, para síntese).

\section{CONCLUSÕES}

A associação de organismos preservados em eventos de alta energia com bivalves que demonstram taxa de erosão acentuada, reflete variações do comportamento energético dos corpos fluviais presentes na Formação Marilia, na região do município de Monte Alto, São Paulo. Épocas de maior afluxo de água do rio podem ter facilitado a preservação da classe tafonômica, onde os organismos estão articulados e fora de posição de vida. Seu soterramento foi rápido e não é descartada a hipótese de grandes fluxos de água característicos de início do verão. Por outro lado, a classe tafonômica que apresenta o truncamento posterior das conchas indica uma provável exposição dessa região da valva a um agente erosivo. Em épocas de seca, os bivalves dulcícolas, pela falta de capacidade de reescavação do substrato, estão suscetíveis a permanecerem próximos à interface água/sedimento e sofrerem uma maior ação erosiva do rio.

O estudo mais acurado dos bivalves da Formação Marília pode vir a complementar o entendimento do funcionamento e relação dos corpos dulcícolas do paleoambiente do Grupo Bauru na região.

\section{AGRADECIMENTOS}

Os autores gostariam de agradecer a A. B. Tiossi, I. H. Zanzini e F. A. Carbonaro, pela ajuda nos trabalhos de coleta de material e de preparo das amostras, assim como os revisores desse artigo que, de maneira salutar, acrescentaram em sua versão final Pesquisa realizada com apoio financeiro da Fundação de Amparo à Pesquisa do Estado de São Paulo (FAPESP) (Processo 05/00506-5).

\section{REFERÊNCIAS}

ARID, F. M., L. P. VIZZOTO \& P. M. P. LANDIN, 1962. A ocorrência de um jazigo fossilífero nos arredores de São José do Rio Preto. Ciência e Cultura 14(3): 172.

ARID, F. M. \& L. D. VIZZOTO, 1963. Sobre vertebrados fósseis no município de Ibirá, São Paulo. Ciência e Cultura 15(3): $181-$ 182.

BATEZELLI, A., A. R. SAAD, M. L. C. ETCHEBEHERE, J. A. J. PERINOTTO \& V. J. FULFARO, 2003. Análise estratigráfica aplicada à Formação Araçatuba (Grupo Bauru - Ks) no Centro-Oeste do Estado de São Paulo. Revista Brasileira de Geociências 22: 5-19.

BATEZELLI, A., A. R. SAAD \& G. BASILICI, 2007. Arquitetura deposicional e evolução da seqüência aluvial neocretácea da porção setentrional da Bacia Bauru, no sudeste brasileiro. Revista Brasileira de Geociências 37(1): 163-181.

CAMPANHA, V. A., M. L. C. ETCHEBEHERE, A. R. SAAD \& V. J. FULFARO, 1993. Novas ocorrências fossilíferas no Grupo Bauru na região do triângulo mineiro, MG, Brasil. Revista Brasileira de Geociências 12(2): 353-372.

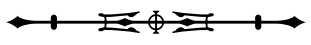


CASTRO, J. C., D. DIAS-BRITO, E. MUSACCHIO, J. SUAREZ, M. S. A. S. MARANHÃO \& R. RODRIGUEZ, 1999. ArcabouçO estratigráfico do Grupo Bauru no oeste paulista, Brasil. Boletim do Simpósio sobre el Cretáceo de América Del Sur e Simpósio sobre o Cretáceo do Brasil 1/5: 509-515.

COIMBRA, A. M. \& L. A. FERNANDES, 1994. A paleogeografia da Bacia Bauru (Cretáceo Superior, Brasil). Actas do Congresso Argentino de Paleontologia y Bioestratigrafia 6: 85-90.

DUARTE, M. M., C. L. SCHIRMER, A. E. C. FREITAS \& C. O. DIEFENBACH, 1996. Habitat selection in freshwater mussels (Mollusca; Bivalvia) in Suzana Lake, Southern Brasil. Biociências $4(2): 17-29$

FERNANDES, L. A. \& A. M. COIMBRA, 1996. A Bacia Bauru (Cretáceo Superior, Brasil). Anais da Academia Brasileira de Ciências 68(2): 195-205.

FULFARO, V. J. \& J. A. J. PERINOTTO, 1996. A Bacia Bauru: Estado da Arte. Boletim do Simpósio sobre o Cretáceo do Brasil 4: 297-303.

FÜRSICH, F. T. \& W. OSCHMANN, 1993. Shell beds as tools in basin analysis: the Jurassic of Kachchh, western India. Journal of Geological Society 150: 169-185.

GHILARDI, R. P. \& M. G. SIMÕES, 2000. Bivalves Fósseis como indicadores da dinâmica sedimentar: um estudo de caso do Paleozóico Superior da Bacia do Paraná, Brasil. Acta Geológica Leopoldensia 23(51): 3-20.

GHILARDI, R. P. \& M. G. SIMÕES, 2002. Foram os bivalves do Grupo Passa Dois (exclusive Formação Rio do Rasto) moluscos dulcícolas? Pesquisas em Geociências 29: 91-92.

GHILARDI, R. P \& G. F. S. BENEDETTI, 2004. Reconhecimento de cicatrizes musculares e considerações prévias sobre a Paleoecologia e Tafonomia de ?Anodontites (Bivalvia, Unionoida) do Grupo Bauru (Cretáceo Superior, Bacia Bauru). Boletim de Resumos da Paleo 12-13.

HUENE, F. VON, 1927. Contribución a la Paleogeografia de Sud América - II - Las Relaciones Paleogeográficas de Sud Geográfica durante el Cretáceo Superior. Academia Nacional de Ciências 30: 256-294.

HUEHNER, M. K., 1987. Field and laboratory determination of substrate preferences of unionid mussels. Ohio Journal of Science 87(1): 29-32.

IHERING, H., 1913. Pleiodon priscus sp.n. In: COMISSÃO GEOGRÁPHICA E GEOLÓGICA. Exploração do Rio Grande e seus afluentes: 1-39. São José dos Dourados, São Paulo.

KIDWELL, S. M., F. T. FÜRSICH \& T. AIGNER, 1986. Conceptual framework for the analysis of fossil concentrations. Palaios 1: 228-238.
KIDWELL, S. M. \& S. M. HOLLAND, 1991. Field description of coarse bioclastic fabrics. Palaios 6: 426-434.

MANZINI, F. F., 1996. O Cretáceo da região de Monte Alto. Revista Geociências 15(2): 123.

MARINHO, T. S., L. C. B. RIBEIRO \& I. S. CARVALHO, 2006. Morfologia de osteodermos de Crocodilomorfos do Sítio Paleontológico de Peirópolis (Bacia Bauru, Cretáceo Superior). Anuário do Instituto de Geociências 29: 42-51.

MEZZALIRA, S., 1966. Os fósseis do Estado de São Paulo. Boletim do Instituto Geográfico e Geológico 45: 1-128.

MEZZALIRA, S., 1974. Contribuição ao conhecimento da estratigrafia e paleontologia do arenito Bauru. Boletim do Instituto Geográfico e Geológico 51: 1-163.

MEZZALIRA, S. \& L. R. L. SIMONE, 1997. A posição sistemática de alguns bivalves Unionoidea do grupo Bauru (Cretáceo Superior) do Brasil. Geociências 2(6): 63-65.

MEZZALIRA, S. \& L. R. L. SIMONE, 1999. Duas novas espécies de Castalia (Mollusca, Bivalvia, Unionoida) no Cretáceo do Grupo Bauru, São Paulo, Brasil. Geociências 4(6): 58-60.

PARODIZ, J. J., 1969. The tertiary non-marine mollusca of South America. Annals of Carnegie Museum 40: 1-242.

PETRI, S. \& V. J. FULFARO, 1983. Geologia do Brasil: Fanerozóico: 1-631. EDUSP, São Paulo.

POPP, J. H. \& J. J. BIGARELLA, 1975. Formações cenozóicas do noroeste do Paraná. Anais da Academia Brasileira de Ciências 47: 465-472.

PRICE, L. I., 1953. Os quelônios da Formação Bauru, Cretáceo terrestre do Brasil meridional. Boletim do Departamento Nacional de Produção Mineral 147: 1-39.

SAVAZZI, E. \& Y. PEIYI, 1992. Some morphological adaptations in freshwater bivalves. Lethaia 25: 195-209.

SENRA, M. C. E. \& L. H. SILVA E SILVA, 1999. Moluscos dulçaqüícolas e microfósseis vegetais associados da Formação Marilia, Bacia Bauru (Cretáceo Superior), Minas Gerais, Brasil. Boletim do Simpósio sobre o Cretáceo do Brasil 5: 497-500.

SIMOES, M. G. \& M. KOWALEWSKI, 1998. Genetic complexity and geobiological implications of simple shell-beds: an example from the upper permian of the Paraná Basin, Brasil. Journal of African Earth Sciences, South Africa 27(1A): 179-180.

SIMÕES, M. G. \& R. P. GHILARDI, 2000. Protocolo tafonômico/ paleoautoecológico como ferramenta nas análises paleossinecológicas: exemplos de aplicação em concentrações fossilíferas do Paleozóico da Bacia do Paraná, Brasil. Revista Pesquisas em Geociências 27(2): 3-13

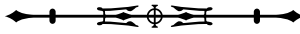


SIMONE, L. R. L. \& S. MEZZALIRA, 1993. Vestígios de partes moles em um bivalve fóssil (Unionoida, Mycetopodidae) do Grupo Bauru (Cretáceo Superior), São Paulo, Brasil. Anais da Academia Brasileira de Ciências 65(2): 155-159.

SIMONE, L. R. L. \& S. MEZZALIRA, 1994. Fóssil Molluscs of Brazil. Boletim do Instituto Geológico 11: 1-202.

STANLEY, S. M., 1970. Relation of shell form to life habits of the Bivalvia (Mollusca). Geological Society of America, Memoirs 125: 1-296.
SUAREZ, J. M. \& M. R. ARRUDA, 1968. Jazigo fossilífero no Grupo Bauru, contendo lamelibrânquios. Anais do Congresso Brasileiro de Geologia 22: 209-212.

SUAREZ, J. M., 1969. Um quelônio da Formação Bauru. Anais do Congresso Brasileiro de Geologia 23: 167-176.

TEVESZ, M. J. S. \& P. L. MCCALL, 1979. Evolution of substratum preference in bivalves (Mollusca). Journal of Paleontology 53: 112-120. 